\title{
The effect of early-life nutrition on DNA repair and DNA methylation in the brain of newborn piglets
}

\author{
S. A. S. Langie ${ }^{1}$, B. Tomaszewski ${ }^{1}$, P. Kowalczyk ${ }^{2,3}$, R. W. L. Godschalk ${ }^{4}$, B. Tudek ${ }^{2,5}$, \\ F. J. van Schooten ${ }^{4}$, R. Zabielski ${ }^{6}$ and J. C. Mathers ${ }^{1}$ \\ ${ }^{1}$ Centre for Brain Ageing and Vitality, Human Nutrition Research Centre, Institute for Ageing and Health, Newcastle \\ University, Newcastle upon TyneNE2 4HH, UK, ${ }^{2}$ Institute of Biochemistry and Biophysics PAS and ${ }^{3}$ Interdisciplinary \\ Centre for Mathematical and Computational Modelling, University of Warsaw, Warsaw, Poland, ${ }^{4}$ Department of Health \\ Risk Analysis and Toxicology, Maastricht University, Maastricht, The Netherlands, ${ }^{5}$ Institute of Genetics and Biotechnology \\ University of Warsaw and ${ }^{6}$ Department of Physiological Sciences, Faculty of Veterinary Medicine, Warsaw University of \\ Life Sciences, Warsaw, Poland
}

\begin{abstract}
Exposure to xenobiotic agents or sub-optimal nutrition during brain development may enhance brain ageing and susceptibility to age-related neurodegenerative disorders. Due to its high utilisation of oxygen and relatively low antioxidants levels, the brain is particularly vulnerable to oxidative stress. The proper functioning of DNA damage responses, e.g. DNA repair, is thus of importance for neuronal survival. Supplementation with antioxidants might up-regulate DNA repair, protecting the brain against genotoxic damage and so reduce neuronal loss associated with ageing. Moreover, environmental factors can lead to changes in DNA methylation status of genes encoding DNA repair enzymes and thus modulate DNA repair capacity and susceptibility to oxidative DNA damage.

We aim to study the role of DNA repair in neuronal cell survival as well as the role of epigenetic mechanisms in mediating the effects of environmental exposures. To investigate the relation between oxidative stress and DNA repair, oxidative stress was induced in newborn piglets by intramuscular injection of $\mathrm{Fe}(200 \mathrm{mg})$ at day 3 after birth. In addition, we investigated the effect of supplementation of pregnant sows with a diet rich in PUFA and antioxidants on the degree of oxidative DNA damage and on the capacity for DNA repair in offspring brain. Piglets were killed at 1, 2, 4, 7, 14 and $28 \mathrm{~d}$ after birth and hippocampus tissues were collected. Raised levels of 8-oxo-7,8-dihydro-2'-deoxyguanosine were detected in the hippocampi of control $v$. supplemented piglets $(P=0.001)$ immediately after birth. Base-excision repair (BER), the major pathway for repair of oxidative DNA damage, was measured using a modified comet assay. BER capacity in the hippocampus of the newborn piglets was not significantly increased in the supplemented group compared with the controls. In addition, BER capacity did not change with age. Next, global DNA methylation was determined by means of a LUminometric Methylation Assay and showed no significant differences between piglets from control and supplemented mothers. However, for individual piglets, there was a significant inverse correlation between BER capacity and DNA methylation $\left(R^{2}=0.133\right.$, $P=0.009)$. We are following this up by investigating changes in promoter methylation patterns for specific BER-related genes using pyrosequencing-based techniques.
\end{abstract}

1. Centre for Brain Ageing and Vitality is funded under the Lifelong Health and Wellbeing cross council initiative by the MRC, BBSRC, EPSRC and ESRC. 